\title{
Technological Advances in Bifidobacterial Molecular Genetics: Application to Functional Genomics and Medical Treatments
}

\author{
Satoru FUKIYA ${ }^{1 *}$, Yosuke HIRAYAMA ${ }^{1}$, Mikiyasu SAKANAKA ${ }^{1}$, Yasunobu KANO $^{2}$ and \\ Atsushi YOKOTA1 \\ ${ }^{1}$ Laboratory of Microbial Physiology, Research Faculty of Agriculture, Hokkaido University, Kita 9 Nishi 9, Kita-ku, Sapporo, Hokkaido \\ 060-8589, Japan \\ 2 Department of Molecular Genetics, Kyoto Pharmaceutical University, 5 Nakauchi-cho, Misasagi, Yamashina-ku, Kyoto 607-8414, \\ Japan
}

Received November 21, 2011; Accepted January 17, 2012

Bifidobacteria are well known as beneficial intestinal bacteria that exert health-promoting effects in humans. In addition to physiological and immunological investigations, molecular genetic technologies have been developed and have recently started to be applied to clarify the molecular bases of host-Bifidobacterium interactions. These technologies include transformation technologies and Escherichia coli-Bifidobacterium shuttle vectors that enable heterologous gene expression. In this context, a plasmid artificial modification method that protects the introduced plasmid from the restriction system in host bifidobacteria has recently been developed to increase transformation efficiency. On the other hand, targeted gene inactivation systems, which are vital for functional genomics, seemed far from being practically applicable in bifidobacteria. However, remarkable progress in this technology has recently been achieved, enabling functional genomics in bifidobacteria. Integrated use of these molecular genetic technologies with omics-based analyses will surely boost characterization of the molecular basis underlying beneficial effects of bifidobacteria. Applications of recombinant bifidobacteria to medical treatments have also progressed.

Key words: Bifidobacterium; molecular genetics; cryptic plasmid; heterologous gene expression; targeted gene inactivation; functional genomics; enzyme/prodrug therapy

\section{INTRODUCTION}

Bifidobacteria were firstly described by Tissier in 1900 , and their health-promoting effects, such as prevention of diarrhea, alleviation of constipation, resistance to microbial infection, possible roles in cancer prevention and in a treatment of inflammatory bowel disease, have been described $[1,2]$. In Japan, many researchers had participated in investigation of the health-promoting functions and intestinal ecology of bifidobacteria under the leadership of Mitsuoka and made tremendous contributions to bifidobacterial research [3, 4]. Development of recombinant DNA techniques in 1970 s and their wide application to bacterial genetics from the 1980 s to 1990 s drove molecular genetics in lactic acid bacteria and bifidobacteria. In addition, starting from the late of

*Corresponding author. Mailing address: Satoru Fukiya, Laboratory of Microbial Physiology, Research Faculty of Agriculture, Hokkaido University, Kita 9 Nishi 9, Kita-ku, Sapporo, Hokkaido 060-8589, Japan. Phone: +81-11-706-2501. Fax: +81-11-706-2501.

E-mail: s-fukiya@chem.agr.hokudai.ac.jp 1990s, genome sequencing of these bacteria has been conducted, and tremendous information about their genomes is currently available. In this review, we would like to focus on the contribution of Japanese researchers to the molecular genetic research in bifidobacteria, especially in regard to works using bifidobacterial cryptic plasmids including not only basic research but also medical applications. In addition, genomics and omics-based research are briefly addressed. We also would like to mention the recent development of gene manipulation tools and their application to functional genomics in bifidobacteria.

\section{BIFIDOBACTERIAL CRYPTIC PLASMIDS AND SHUTTLE VECTORS}

Bifidobacterial cryptic plasmids were first identified and investigated in the 1980's [5-8]. As one of the early works, Iwata and Morishita investigated the presence of the plasmids in forty-two strains of Bifidobacterium breve [8]. In contrast to another work in which no cryptic plasmids were found among investigated $B$. breve strains 
[5], approximately $40 \%$ (18 strains) of the $B$. breve strains were revealed to possess the plasmids. These strains were distinguished into five different plasmid profiles according to the electrophoretic migration patterns of plasmid DNA. Identification of $B$. breve plasmids was a promising finding for development of Bifidobacterium shuttle vectors, although we had to wait for identification of $\mathrm{pNBb} 1$ from $B$. breve ATCC 15698 [9], from which several shuttle vectors were developed [10]. These vectors were basically composed of an $E$. coli plasmid region (from pACYC177 or pUC118), erythromycin-resistance gene and $\mathrm{pNBb} 1$ region for plasmid replication in bifidobacteria. Interestingly, plasmid propagation in Dam and Dcm methylasenegative $E$. coli strains affected the transformation efficiencies of Bifidobacterium strains [10]. Generally, a lack of methylation of plasmid DNA is expected to decrease transformation efficiency, whereas in this case, the effects varied from strain to strain: the shuttle vector $\mathrm{pBE} \Delta 5$ from the methylase-negative $E$. coli showed higher transformation efficiency in some Bifidobacterium strains but showed lower efficiency in other strains. These differences might not only have been due to the lack of plasmid DNA methylation in $E$. coli but might also have been due to the difference in host restriction systems among the Bifidobacterium strains used. The improved vector $\mathrm{pBE} \Delta 4 *$ was successfully used for genetic analysis of the rpsL gene encoding ribosomal protein S12 to clarify its role in streptomycin resistance in $B$. breve strain Yakult [11].

Beginning in the late 1990's, Kano and his colleagues developed several E. coli-Bifidobacterium shuttle vectors [12-14]. These vectors were based on a cryptic plasmid pTB6 (3.6-kbp) in Bifidobacterium longum BK51 [12]. The plasmid pTB6 was predicted to replicate in the rolling-circle replication manner based on the structural similarity to a known B. longum plasmid, pKJ36 [15]. In combination with the highly transformable strain B. longum 105-A, pTB6-based shuttle vectors showed relatively high transformation efficiency. For instance, pBLES100 (9.1-kbp) and pDSO44Sp (4.3-kbp) showed transformation efficiencies of $1.6 \times 10^{4}$ and $1.3 \times 10^{6} \mathrm{cfu} / \mu \mathrm{g}$ DNA, respectively $[12,13]$. In addition, pKKT427 (3.9-kbp), the smallest derivative of pTB6-derived shuttle vectors, showed a transformation efficiency of $1.0 \times 10^{6}$ to $3.0 \times 10^{6} \mathrm{cfu} / \mu \mathrm{g}$ DNA [14]. Development of these vectors contributed to further molecular genetic research in bifidobacteria as mentioned below.

\section{HETEROLOGOUS GENE EXPRESSION IN BIFIDOBACTERIA AND ITS USE FOR MEDICAL TREATMENT}

Kano and his colleagues also identified the hup gene, encoding the histone-like protein $\mathrm{HU}$, in $B$. longum ATCC 15707 [16]. Expression of hup from its native upstream regulatory region restored the growth of $\mathrm{Mu}$ phage in integration host factor-deficient $E$. coli, which indicated that the native regulatory region of hup works in both E. coli and B. longum [16]. It was also indicated that the expression level of native hup in B. longum was high and comparable to that of $E$. coli, which suggested that the regulatory region of hup is useful for gene expression in B. longum [17]. These observations led to attempts to express an exogenous gene in $B$. longum using the regulatory region of hup. These works were conducted by Taniguchi and his colleagues with Kano.

The first example of heterologous expression using the hup-regulatory region was E. coli cytosine deaminase (CD) [18]. The promoter region and 5' region of hup ORF was fused in-frame to the CD gene and cloned into the shuttle vector pBLES100. CD was successfully expressed in B. longum 105-A, which was confirmed by the detection of $\mathrm{CD}$ by $\mathrm{CD}$-specific antibodies and by quantification of the conversion of 5-fluorocytosine (5FC) to 5-fluorouracil (5-FU) [18]. This research was aimed at establishing an enzyme/prodrug therapy for solid tumors using 5-FU as an anticancer agent. Administration of a large dose of 5-FU into the whole body is necessary to achieve a sufficient local concentration of 5-FU around a tumor, although 5-FU is toxic to normal growing cells and has various side effects. In contrast, 5-FC is less toxic than 5-FU. Specific localization and growth of Bifidobacterium bifidum were also observed in tumors after intravenous administration in mice [19]. This phenomenon is due to the low oxygen partial pressure inside of solid tumors, which is a preferable environment for anaerobic bifidobacteria. These observations and successful expression of $C D$ in $B$. longum 105-A led to establishment of the concept of using recombinant $B$. longum cells to achieve an effective local concentration of 5-FU around tumors by local conversion of low-toxic 5-FC to 5-FU.

Based on these findings, Taniguchi et al. began a series of studies related to this concept. Localized growth of wild-type and recombinant B. longum 105-A harboring pBLES100 was demonstrated in transplanted cancer tumors generated by inoculation of cultured cancer cells such as B16-F10 melanoma cells and Lewis lung cancer cells in mice [20]. Localization of these strains was also verified in chemically-induced 
mammary tumors in rats [21]. Subsequently, B. longum 105-A harboring pBLES100-S-eCD that expressed recombinant $\mathrm{CD}$ was used in a trial enzyme/prodrug therapy for autochthonous mammary tumors in rats [22]. Selective localization of $B$. longum in the tumor and tumor-specific conversion of 5-FC to $5-\mathrm{FU}$ by the recombinant $B$. longum were observed. As a consequence of the tumor-specific conversion, suppression of tumor growth by the introduction of recombinant $B$. longum via intravenous injection was observed. Surprisingly, antigenic tests of recombinant $B$. longum in guinea pigs showed that the recombinant $B$. longum has little antigenicity, with the level being as low as that of saline. Further improvement of CD activity [23] and expression of $\mathrm{CD}$ in another species, B. breve [24], were also achieved.

The success of the enzyme/prodrug therapy for solid tumors with the CD-expressing B. longum has opened the way to use of recombinant bifidobacteria for medical treatments. Currently reported studies aiming at medical treatments using recombinant bifidobacteria are listed in Table 1. These include not only cancer therapies [25-27] but also the production of recombinant human hormone [28], therapeutic proteins [29, 30], and protective antigens for infectious diseases [31, 32]. As indicated in Table 1, the hup regulatory region has become the first choice for the expression of heterologous genes in bifidobacteria [18, 22-24, 29-31], and B. longum 105-A has been frequently used for these studies because it has a relatively high transformation efficiency [12]. It is remarkable that recombinant $B$. longum 105-A expressing Salmonella flagellin on the cell surface was used as an oral vaccine that successfully protected mice from a challenge with Salmonella Typhimurium [32]. In this work, the antigen was successfully expressed with the aid of a bifidobacterial glt $A$ gene encoding the galacto- $N$-biose/lacto- $N$-biose I-binding protein, GL-BP, a component of the ATP-binding cassette (ABC) transporter for these disaccharides. Because GL-BP was thought to be anchored to the cell membrane by the Nterminal lipid modification and the C-terminal of this protein had structural flexibility [33], in-frame fusion of the flagellin gene to the 3'-terminus of gltA allowed surface expression of Salmonella flagellin [32].

Because of their harmless and less antigenic nature in humans, recombinant bifidobacteria will be widely used in various fields of medical treatment.

\section{HETEROLOGOUS GENE EXPRESSION IN BIFIDOBACTERIA FOR FUNCTIONAL CHARACTERIZATION OF BIFIDOBACTERIAL GENES FOR DEGRADATION OF MUCIN GLYCOPROTEINS AND HUMAN MILK OLIGOSACCHARIDES}

Yamamoto, Kitaoka and their colleagues have reported various kinds of novel bifidobacterial glycosidases and related proteins for utilization and metabolism of sugar chains of host mucin glycoproteins and human milk oligosaccharides (HMOs) [reviewed in 34 and 35]. These works revealed the importance of the ability to degrade sugar chains of mucin for bifidobacterial colonization in the human large intestine and HMOs for establishment of dominance of several Bifidobacterium species in the large intestine of breastfed infants.

Among their works, heterologous gene expression in bifidobacteria was used to characterize the physiological roles of the three glycosidases in Bifidobacterium bifidum JCM 1254, namely, 1,2- $\alpha$-L-fucosidase (AfcA), 1,3-1,4- $\alpha$-L-fucosidase (AfcB) [36] and exo- $\alpha$-sialidase (SiaBb2) [37]. B. longum 105-A was used as an expression host because it has no endogenous $\alpha$-Lfucosidase and exo- $\alpha$-sialidase activities. Modified shuttle vectors derived from pBLES100 [12] were used for the introduction of genes encoding these enzymes to B. longum 105-A. Fucose was revealed to be liberated from substrate 2'-fucosyllactose by AfcA and from substrates 3-fucosyllactose and lacto- $N$-fucopentaose II by $\mathrm{AfcB}$, respectively. These results indicated that AfcA and $A f c B$ are essential for the utilization of fucosylated HMOs [36]. SiaBb2 was revealed to degrade sialyloligosaccharides such as 3'-sialyllactose and 6'sialyllactose, indicating its critical importance in the utilization of sialylated oligosaccharides in HMOs [37].

\section{GENOMICS- AND OMICS-BASED STUDIES}

Since the first description of the complete genome sequence of B. longum NCC2705 in 2002 [38], many genomic sequences have been determined in bifidobacteria. Although the detailed characteristics of genomes in each species and information from comparative genomics are beyond our scope [reviewed in 39-41], it should be noted that Japanese researchers also contributed to the progress in this field. For example, the genomes of the following strains have been completely sequenced: Bifidobacterium adolescentis ATCC 15703 (Suzuki et al., GenBank accession No. AP009256), B. longum subsp. longum JCM $1217^{\mathrm{T}}$ and $B$. longum subsp. infantis $157 \mathrm{~F}$ [42] and commercially used 


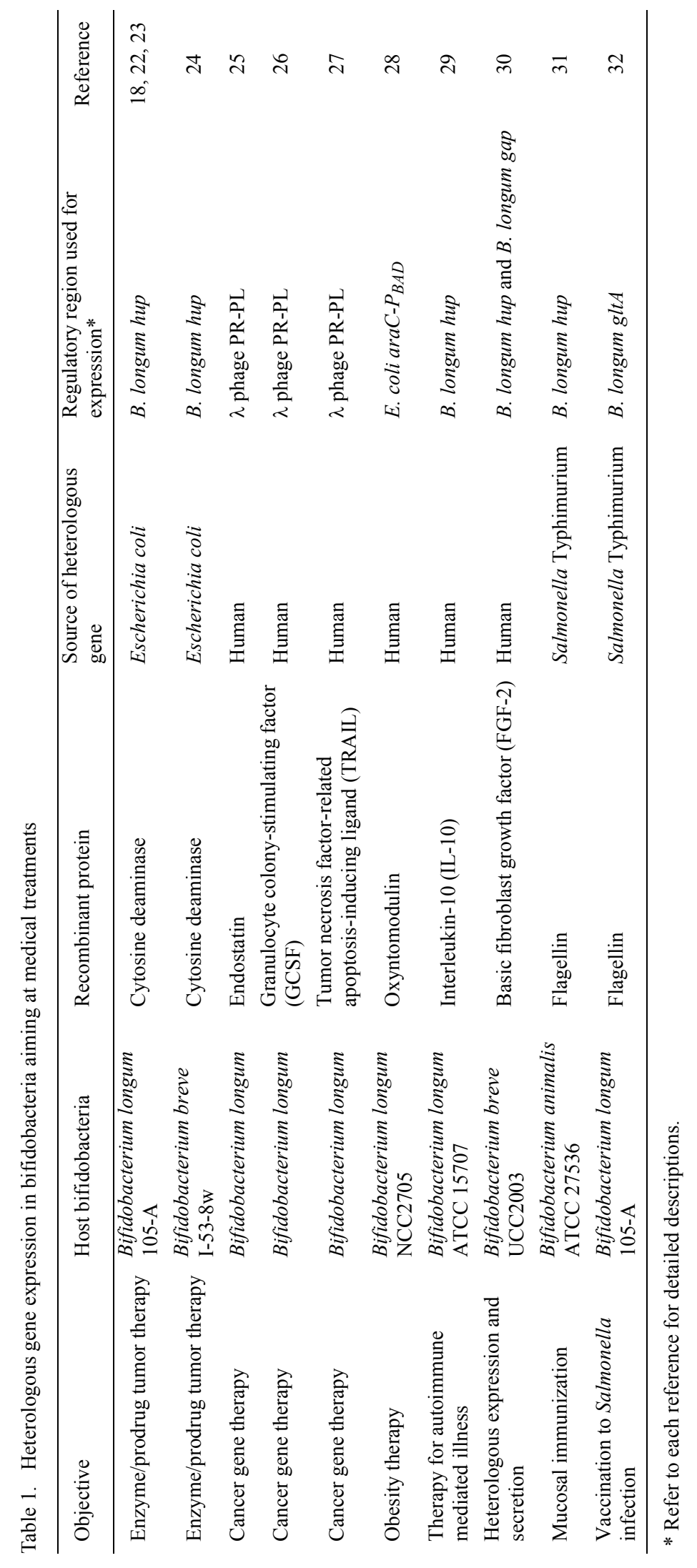


strains B. breve strain Yakult [43] and B. longum BB536 [44]. Here, we would like to describe examples of genomics- and omics-based studies.

Based on the genomic sequence of $B$. adolescentis ATCC 15703, a tiling array was prepared and used for a comparative genomic hybridization $(\mathrm{CGH})$ analysis among $B$. adolescentis strains to investigate the intraspecies diversity [45]. This CGH analysis revealed that the intraspecies genomic differences were point mutations and gene deletions. Representative absent genes in the genomes of tested strains fell into the cluster of orthologous group (COG) category M (cell wall/ membrane/envelope biogenesis) and category $G$ (carbohydrate transport and metabolism), suggesting the involvement of these genes in the host-bifidobacterial specificity.

A similar intraspecies comparison using polymorphism analysis of ORFs encoding putative cellsurface adhesins in 42 strains of B. longum subsp. longum revealed that an ORF named BL0675 encoding putative fimbrial subunit protein had a highly variable sequence in each strain [46]. These sequences were classified into five variant types based on the sequence polymorphisms. The detection frequency of these variant types in human feces was also investigated by a quantitative PCR method using specific primers for each variant type. It was inferred that BL0675 might contribute to host-specific colonization as a fimbrial protein with glycoprotein-binding ability [46].

Screening of candidate genes involved in mucosal cross talk was recently reported by Ishikawa et al. [47]. It was hypothesized that the beneficial effects of bifidobacteria are attributed to induction of the genes involved in mucosal cross talk in the gastrointestinal tract. As the first step to verify their hypothesis, candidate genes of $B$. breve strain Yakult (BbrY) implicated in mucosal cross talk were screened. BbrY was administered to germ-free mice, and the contents of the cecum on days 3 or 28 after administration were used for the BbrY transcriptome analyses. Tripartite comparison of BbrY transcriptomes among transcriptomes in the cecal contents and those in in vitro m-ILS cultures was conducted by microarray experiments. Thereafter, 93 candidate genes upregulated in the mouse intestine were identified through clustering analyses [47]. These candidate genes might include genes responding only to the intestinal environment, such as to a lack of nutrition and osmotic pressure, which may not be involved in the mucosal cross talk. Therefore, BbrY transcriptomes in in vitro fecal cultures, which mimic nutritional conditions in the intestine, were compared with those in m-ILS cultures by microarrays to identify the genes responding only to the intestinal environment. Genes upregulated in both the mouse intestine and the fecal cultures were excluded from the candidate genes because these genes were assumed not to be involved in the mucosal cross talk. Consequently, 45 genes were classified as upregulated specifically in the mouse intestine. Most of those genes were involved in sugar transport (e.g., ABC transporters and the phosphotransferase system) and sugar liberation (e.g., glycosidases). These results suggest that BbrY liberates sugar from mucin or residual dietary components and then incorporates them into the cells to maintain energy homeostasis. Free sugars might also be liberated from sugar chains on the epithelium by glycosidases. In addition, genes for acetate production via pyruvate were also upregulated. As acetate is an important energy source for epithelial cells, the supply of acetate through the fermentation of liberated sugars also seems important cross talk that is beneficial to their hosts.

\section{TECHNOLOGICAL DEVELOPMENT FOR FUNCTIONAL GENOMICS}

\section{Plasmid artificial modification method}

Despite the development of numerous E. coliBifidobacterium shuttle vectors [reviewed in 48 and 49], the generally low transformation efficiency, with a median of up to $10^{3} \mathrm{cfu} / \mu \mathrm{g}$ DNA for the efficiency of developed host-vector systems [49], has prevented the progress of functional gene analysis. Several methodological trials for improvement of transformation efficiency have been conducted such as addition of highosmotic solutes into the washing buffer for preparation of competent cells and optimization of electroporation conditions. These improvements are valuable but do not seem to be crucial for the strains with very low transformation efficiency. Suzuki and colleagues made the first breakthrough concerning this issue [14]. They hypothesized that the low transformation efficiency in these strains is mainly due to the degradation of foreign DNA by the bifidobacterial restriction system. This hypothesis seemed in agreement with the other observations indicating that a shuttle vector purified from already transformed bifidobacterial strains produced a higher transformation efficiency than the same vector prepared from $E$. coli $[50,51]$. Therefore, putative type II DNA methyltransferase genes in the genome of $B$. adolescentis ATCC 15703 were expressed in $E$. coli, and shuttle vector pKKT427 was modified in 
this $E$. coli by these methyltransferases. After the modification, this vector produced elevated transformation efficiency ( $10^{5} \mathrm{cfu} / \mu \mathrm{g}$ DNA). It was five orders of magnitude higher than that achieved by the same vector prepared from the parental $E$. coli host. This approach was called plasmid artificial modification (PAM). The PAM approach was also effective in Lactococcus lactis IO-1 as a transformation host. A similar strategy was introduced to improve the transformation efficiency in B. breve UCC2003, which allowed vector insertion into the chromosomal genes [52]. Although the PAM approach requires genomic information to identify methylase genes of a given bifidobacterial strain, increasing availability of genomic information of many bifidobacterial strains will surely facilitate the application of this approach to various bifidobacterial strains.

\section{Targeted gene inactivation by homologous recombination}

Targeted gene inactivation had long been impossible in bifidobacteria, although the technique is essential for functional gene analysis. Currently, at least three different strategies are available for targeted gene inactivation in bifidobacteria, as shown in Figure 1. Here, we would like to describe the fundamental concept and characteristics of each strategy.

a) Single-crossover plasmid insertion

This strategy is achieved by plasmid insertion into a target gene via a single crossover (Fig. 1A). As the first step, an internal DNA fragment of the target gene is cloned into the targeting vector. This vector is basically nonreplicative in the host Bifidobacterium strain. After introduction of the targeting vector into the host, homologous recombination can take place between the cloned fragment and chromosomal target gene (a first crossover). This ultimately results in splitting of the target gene by the inserted vector (Fig. 1A). The first example of the application of this strategy in bifidobacteria was the inactivation of the $a p u B$ gene encoding amylopullulanase in $B$. breve UCC2003 [53]. In this case, a combination of the plasmids allowing conditional replication of the nonreplicative gene targeting vector pORI19 was used to increase the chance of homologous recombination. Although the plasmid insertion mutant of $a p u B$ was successfully constructed, this method seemed laborious and time-consuming. Therefore, this method was improved and simplified by the approach for escaping from the barrier of the bifidobacterial DNA restriction system [52]. Modification of pORI19 in E. coli expressing methylase genes from the $B$. breve $\mathrm{UCC} 2003$ genome resulted in an increase in transformation efficiency, leading to insertion of pORI19 into the target genes in B. breve UCC2003 without using the conditional replication strategy described above. Consequently, plasmid insertion inactivation became available by a one-round transformation of the targeting plasmid. This simplified method was also used for inactivation of the gene encoding cellodextrin-binding protein CldE [54] and ATPase for assembly of type IVb tight adherence (Tad) pili in $B$. breve UCC2003 [55].

b) Double-crossover gene disruption

This strategy is achieved by double crossover (Fig. 1B). The mutated allele of the target gene, which is generated by the introduction of a marker gene (e.g., antibiotic resistance gene) into the target allele, is cloned into a nonreplicative targeting vector. Introduction of the targeting vector with mutated allele into the host cell results in integration of the vector into the target locus by homologous recombination (a first crossover). The first crossover can take place at either of the homologous regions flanking the marker gene (Fig. 1B, region $\mathrm{X}$ or Y). Subsequently, if the second crossover occurs between the homologous region in the vector and a chromosome in a different region than that used in the first crossover, replacement of the wild-type allele in the chromosome with the mutated one having a marker gene will result (allelic exchange), generating the desired gene disruptant. This strategy was first applied in bifidobacteria for inactivation of an ABC-type carbohydrate transporter gene, BL0033, in B. longum subsp. longum NCC2705 [42].

c) Double-crossover markerless gene deletion

Double crossover was applied to introduction of markerless gene deletion in the bifidobacterial chromosome (Fig. 1C). The strategy is a patented method by Arigoni and Delley [56]. The flow of the strategy is basically the same as that described for double-crossover gene disruption, except that there are several differences. An E. coli plasmid harboring an antibiotic resistance gene is used as a targeting vector by cloning of adjacent regions of the target gene. The targeting vector is introduced into a bifidobacterial strain, and antibiotic selection of the transformants results in identification of the chromosomal integrants of the targeting vector. After subculturing the integrant for 100 generations without antibiotic selection, the secondcrossover recombinants appear in the subcultured firstcrossover integrants. Antibiotic-sensitive strains that are expected to lose the integrated vector from the chromosome by the second crossover can be identified 


\section{A. Single-crossover plasmid insertion}

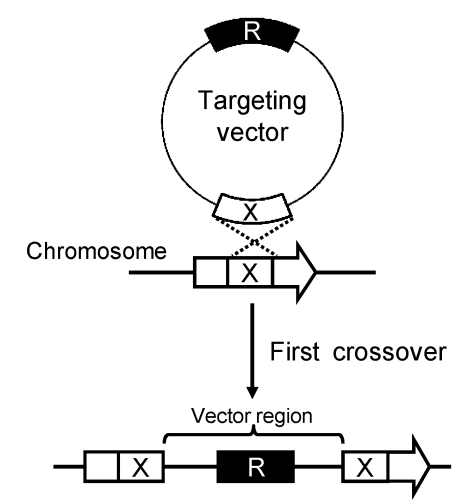

Plasmid insertion mutant

\section{B. Double-crossover gene disruption}

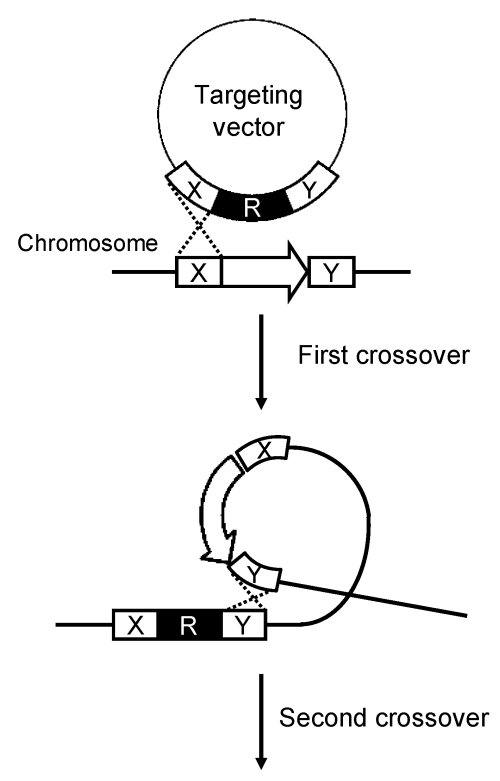

\section{Double-crossover markerless gene deletion}

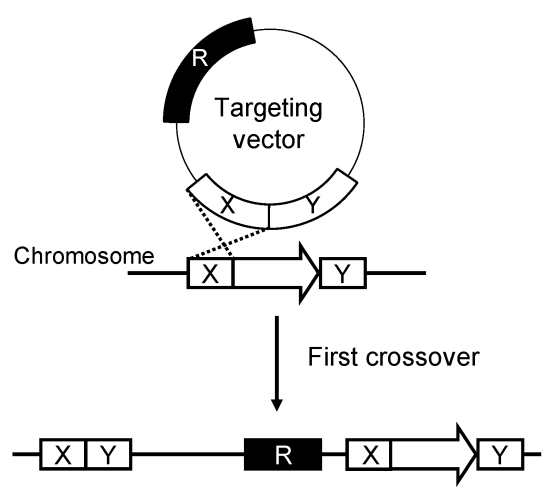

Long-term

subculture

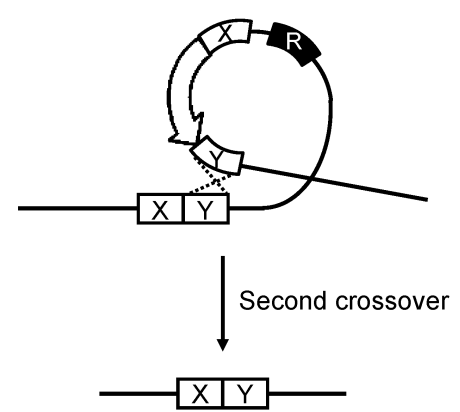

Markerless gene deletion mutant

Gene disruption mutant

Fig. 1. Schematic representation of the targeted gene inactivation methods currently available in bifidobacteria. Target genes for inactivation are indicated as open arrows. Antibiotic resistance genes are represented as filled squares marked with the letter R. In B and $\mathrm{C}$, theoretically, two types of target gene alleles can be generated in both the first crossover and second crossover. However, for simplicity, only the scheme yielding the desired mutant is described. (A) Single-crossover plasmid insertion. A nonreplicative targeting vector harboring the internal region of the target gene (represented as open squares marked with the letter $\mathrm{X}$ ) is used for the first crossover. Homologous recombination between the internal region in the vector and the chromosomal target gene results in splitting of the target gene. (B) Double-crossover gene disruption. A nonreplicative targeting vector harboring the 5' and 3' regions of the target gene (represented as open squares marked with the letters $\mathrm{X}$ and $\mathrm{Y}$ ) between which an antibiotic resistance gene is inserted is used for the first crossover. The first crossover occurred between the homologous regions (in this case X), resulting in insertion of the targeting vector into the target gene. If the second crossover occurs between homologous regions different from those used in the first-crossover recombination (in this case Y), the desired gene disruption mutant will be generated. (C) Doublecrossover markerless gene deletion. The procedure is similar to that indicated in B. However, a nonreplicative targeting vector that harbors $5^{\prime}$ and $3^{\prime}$ regions of the target gene (represented as open squares marked with the letters $\mathrm{X}$ and $\mathrm{Y}$ ) is used for the first crossover. The second crossover can occur during long-term subculture (e.g., 100 generations) of the first-crossover integrants. A markerless gene deletion mutant can be generated when the second crossover occurs as described in B. 
by the replica plating method. Actually, when the strategy was used for deletion of the tet $W$ gene that confers tetracycline resistance in Bifidobacterium animalis subsp. lactis NCC 2818, 163 out of 750 colonies $(21 \%)$ were identified as second-crossover recombinants by replica plating of the first-crossover integrants subcultured for 100 generations. In addition, two tet $W$-deletion strains were successfully identified among the 135 second-crossover recombinants by colony PCR [56].

For introduction of targeted gene inactivation to a given Bifidobacterium strain, several points should be taken into consideration. Among the strategies mentioned above, strategy a, single-crossover plasmid insertion, is the easiest because it requires only one round of transformation procedure. However, it should be noted that the mutated allele in the plasmid-integrated mutant is principally unstable, because the inserted plasmid might be excised by a subsequent crossover that can occur between the homologous regions left in the target allele. Both strategy b, double-crossover gene disruption, and strategy c, double-crossover markerless gene deletion, can introduce stable mutation into the target gene because no homologous region is left in the target locus after the double crossover. Time-consuming screening of the double-crossover mutant is necessary unless a counter selection marker (e.g., Bacillus subtilis $s a c B$, which induces lethality in sucrose-containing medium in other bacteria) or conditional replication plasmid (e.g., thermosensitive replication plasmid) is applied. The first two strategies leave the vector DNA (strategy a) or a marker gene (strategy b) in the mutated gene allele. Maintenance of these exogenous DNA regions occasionally affects the gene expression of surrounding genes of the target locus, leading to confusing results in phenotype analysis of the mutant. It also restricts the introduction of multiple mutations in the same strain due to the limited availability of the antibiotic resistance genes in bifidobacteria. Strategy c can overcome these problems. Because time-consuming and rather inefficient procedures are required for isolation of the double-crossover recombinants and objective gene deletion mutants in this strategy, we have developed an improved method for the strategy c. It was achieved by facilitation of a second-crossover step and efficient elimination of the excised plasmid from the cells. Facilitation of a second-crossover step is accomplished using plasmid replication protein RepA, and efficient elimination of the excised plasmid is accomplished using plasmid incompatibility [Hirayama et al., manuscript in preparation, presented at the Tenth
Symposium on Lactic Acid Bacteria (Poster F027), Egmond aan Zee, The Netherlands, August 28 to September 1, 2011].

In any of the above strategies, high transformation efficiency is required to achieve the first crossover because nonreplicative or conditionally replicative vectors are employed. Therefore, improvement of the transformation efficiency by the PAM method [14] will contribute to the application of these inactivation strategies to bifidobacterial strains, especially with low transformation efficiencies.

\section{FUNCTIONAL GENOMICS IN BIFIDOBACTERIA}

Development of tools for functional gene analysis and introduction of genomics- and omics-based approaches in addition to the basic biochemical and physiological analyses now leads researchers to conduct integrated, functional genomics in bifidobacteria. Two outstanding reports recently published clearly indicated the importance of targeted gene inactivation in clarification of the function of the candidate genes involved in the beneficial effects of bifidobacteria on hosts.

Fukuda et al. [42] revealed the mechanism of bifidobacteria in protection of mice from enterohemorrhagic E. coli O157:H7 (E. coli O157) infection. A significantly low concentration of Shiga toxin 2 (Stx2), a crucial factor in the lethal infection of $E$. coli $\mathrm{O} 157$ strain, was observed in the serum of mice in which the protective $B$. longum strain was administered prior to infection with $E$. coli $\mathrm{O} 157$ as compared with that detected in the serum of mice administered a nonprotective Bifidobacterium strain. Integrated omics approaches revealed that the amounts of acetate produced from carbohydrate fermentation by the Bifidobacterium strains positively correlated with the resistance of mice to the $E$. coli $\mathrm{O} 157$ infection. Then comparative genomics of both protective and nonprotective Bifidobacterium strains were conducted, and it was revealed that two loci encoding ABC-type sugar transporters were specific to the preventive strains. Of these, BL0033-BL0036 were suggested to be involved in preventing the mice from the mortal effect of E. coli O157. A gene knockout mutant of the BL0033 was constructed by inactivation strategy $b$, doublecrossover gene disruption. The knockout mutant was not able to protect mice from the lethal effect of $E$. coli $\mathrm{O} 157$ infection due to a reduced concentration of acetate in fecal samples. These results strongly indicated that increased production of acetate by the protective Bifidobacterium strain contributes to inhibition of the translocation of Stx2 from inside of the gut to the blood, 
resulting in protection of the mice from the mortal effect of $E$. coli $\mathrm{O} 157$ infection.

O'Connell Motherway et al. [55] described an important host-colonization factor in B. breve UCC2003. This factor is the type IVb tight adherence (Tad) pili that are known to be involved in colonization and host pathogenicity in pathogenic bacteria. The tad locus was conserved not only in the $B$. breve $\mathrm{UCC} 2003$ genome but also in $B$. breve strains tested and other known sequenced bifidobacterial genomes. In vivo transcriptome analysis of $B$. breve UCC 2003 was performed in a murine colonization model, and the expression of a type $\mathrm{IVb}$ Tad pilus-encoding gene cluster $\left(\operatorname{tad}_{2003}\right.$ : Bbr_0132-Bbr_0138) was upregulated in the mouse intestine compared with that under in vitro culture conditions. To establish whether $\operatorname{tad}_{2003}$ was involved in colonization of $B$. breve UCC2003, a tadA gene (Bbr_0133, encoding ATPase) mutant was constructed by inactivation strategy a, single-crossover plasmid insertion. Wild-type and mutant strains could colonize in conventional mice at similar levels in the beginning (after 3 days), whereas the colonization level of the tadA mutant was 70-fold lower than that of the wild-type strain at 8 days after administration. Interestingly, both strains maintained colonization at a similar level for three weeks after administration in germ-free mice probably because no competing microbiota were present in the monoassociated mice. Subsequent transfer of monoassociated mice to a conventional area resulted in shedding of tadA mutant 7 days after transfer, and the tadA mutant became undetectable in feces two weeks after transfer in contrast to continued detection of the wild-type strain two weeks after transfer. These results demonstrated that the $\operatorname{tad}_{2003}$ locus is crucially important for colonization and persistence of $B$. breve $\mathrm{UCC} 2003$ as part of the competing gastrointestinal tract microbiota.

\section{CONCLUSION}

In addition to the genomic sequences, molecular genetic tools such as shuttle vectors, gene expression systems and targeted gene inactivation systems are now available in bifidobacteria. Tools that are currently unavailable, such as random mutagenesis using mobile genetic elements including endogenous insertion sequences, should be developed in the future. Refinement of target gene inactivation tools using thermosensitive plasmids or counter selection markers is also necessary. Future studies of bifidobacteria using these molecular genetic tools will be mainly focused on the molecular basis of host-microbe interactions that exert beneficial effects on the host, which have been largely unexplored. It is expected that development of various genetic tools for bifidobacteria will boost these study area as well as the application of recombinant bifidobacteria to various kinds of medical treatments.

\section{REFERENCES}

1. Leahy SC, Higgins DG, Fitzgerald GF, van Sinderen D. 2005. Getting better with bifidobacteria. J Appl Microbiol 98: 1303-1315.

2. Kleerebezem M, Vaughan EE. 2009. Probiotic and gut lactobacilli and bifidobacteria: Molecular approaches to study diversity and activity. Annu Rev Microbiol 63: 269-290.

3. Mitsuoka T, Kaneuchi C. 1977. Ecology of the bifidobacteria. Am J Clin Nutr 30: 1799-1810.

4. Mitsuoka T. 1990. Bifidobacteria and their role in human health. J Ind Microbiol 6: 263-268.

5. Sgorbati B, Scardovi V, Leblanc DJ. 1982. Plasmids in the genus Bifidobacterium. J Gen Microbiol 128: 2121-2131.

6. Sgorbati B, Scardovi V, Leblanc DJ. 1986. Related structures in the plasmid profiles of Bifidobacterium longum. Microbiologica 9: 415-422.

7. Sgorbati B, Scardovi V, Leblanc DJ. 1986. Related structures in the plasmid profiles of Bifidobacterium asteroides, B. indicum and B. globosum. Microbiologica 9: 443-456.

8. Iwata M, Morishita T. 1989. The presence of plasmids in Bifidobacterium breve. Lett Appl Microbiol 9: 165168.

9. Bourget N, Simonet JM, Decaris B. 1993. Analysis of the genome of the five Bifidobacterium breve strains: plasmid content, pulsed-field gel electrophoresis genome size estimation and $r r n$ loci number. FEMS Microbiol Lett 110: 11-20.

10. Iino T, Morishita T. 2007. Shuttle vector for bifidobacteria and replication protein gene of bifidobacteria plasmid. Japanese Patent, JP0003946300B2.

11. Kiwaki M, Sato T. 2009. Antimicrobial susceptibility of Bifidobacterium breve strains and genetic analysis of streptomycin resistance of probiotic $B$. breve strain Yakult. Int J Food Microbiol 134: 211-215.

12. Matsumura H, Takeuchi A, Kano Y. 1997. Construction of Escherichia coli-Bifidobacterium longum shuttle vector transforming B. longum 105-A and 108-A. Biosci Biotechnol Biochem 61: 12111212.

13. Tanaka K, Samura K, Kano Y. 2005. Structural and functional analysis of pTB6 from Bifidobacterium longum. Biosci Biotechnol Biochem 69: 422-425.

14. Yasui K, Kano Y, Tanaka K, Watanabe K, ShimizuKadota M, Yoshikawa H, Suzuki T. 2009. Improvement of bacterial transformation efficiency using plasmid artificial modification. Nucleic Acids 
Res 37: e3.

15. Park MS, Shin DW, Lee KH, Ji GE. 2000. Characterization of plasmid pKJ36 from Bifidobacterium longum and construction of an E. coliBifidobacterium shuttle vector. J Microbiol Biotechnol 10: 312-320.

16. Takeuchi A, Matsumura H, Kano Y. 2002. Cloning and expression in Escherichia coli of a gene, hup, encoding the histone-like protein HU of Bifidobacterium longum. Biosci Biotechnol Biochem 66: 598-603.

17. Goshima N, Kano Y, Imamoto F. 1990. Characterization of $\mathrm{HU}-\mathrm{lik}$ e protein from Bifidobacterium longum. Biochimie 72: 207-212.

18. Nakamura T, Sasaki T, Fujimori M, Yazawa K, Kano Y, Amano J, Taniguchi S. 2002. Cloned cytosine deaminase gene expression of Bifidobacterium longum and application to enzyme/pro-drug therapy of hypoxic solid tumors. Biosci Biotechnol Biochem 66: 23622366.

19. Kimura NT, Taniguchi S, Aoki K, Baba T. 1980. Selective localization and growth of Bifidobacterium bifidum in mouse tumors following intravenous administration. Cancer Res 40: 2061-2068.

20. Yazawa K, Fujimori M, Amano J, Kano Y, Taniguchi S. 2000. Bifidobacterium longum as a delivery system for cancer gene therapy: Selective localization and growth in hypoxic tumors. Cancer Gene Ther 7: 269274.

21. Yazawa K, Fujimori M, Nakamura T, Sasaki T, Amano J, Kano Y, Taniguchi S. 2001. Bifidobacterium longum as a delivery system for gene therapy of chemically induced rat mammary tumors. Breast Cancer Res Treat 66: $165-170$.

22. Sasaki T, Fujimori M, Hamaji Y, Hama Y, Ito K, Amano J, Taniguchi S. 2006. Genetically engineered Bifidobacterium longum for tumor-targeting enzymeprodrug therapy of autochthonous mammary tumors in rats. Cancer Sci 97: 649-657.

23. Hamaji Y, Fujimori M, Sasaki T, Matsuhashi H, Matsui-Seki K, Shimatani-Shibata Y, Kano Y, Amano J, Taniguchi S. 2007. Strong enhancement of recombinant cytosine deaminase activity in Bifidobacterium longum for tumor-targeting enzyme/ prodrug therapy. Biosci Biotechnol Biochem 71: 874883.

24. Hidaka A, Hamaji Y, Sasaki T, Taniguchi S, Fujimori M. 2007. Exogeneous cytosine deaminase gene expression in Bifidobacterium breve I-53-8w for tumor-targeting enzyme/prodrug therapy. Biosci Biotechnol Biochem 71: 2921-2926.

25. Xu YF, Zhu LP, Hu B, Fu GF, Zhang HY, Wang JJ, Xu GX. 2007. A new expression plasmid in Bifidobacterium longum as a delivery system of endostatin for cancer gene therapy. Cancer Gene Ther 14: 151-157.
26. Zhu LP, Yin Y, Xing J, Li C, Kou L, Hu B, Wu ZW, Wang JJ, Xu GX. 2009. Therapeutic efficacy of Bifidobacterium longum-mediated human granulocyte colony-stimulating factor and/or endostatin combined with cyclophosphamide in mouse-transplanted tumors. Cancer Sci 100: 1986-1990.

27. Hu B, Kou L, Li C, Zhu LP, Fan YR, Wu ZW, Wang JJ, Xu GX. 2009. Bifidobacterium longum as a delivery system of TRAIL and endostatin cooperates with chemotherapeutic drugs to inhibit hypoxic tumor growth. Cancer Gene Ther 16: 655-663.

28. Long RT, Zeng WS, Chen LY, Guo J, Lin YZ, Huang QS, Luo SQ. 2010. Bifidobacterium as an oral delivery carrier of oxyntomodulin for obesity therapy: inhibitory effects on food intake and body weight in overweight mice. Int J Obes 34: 712-719.

29. Escogido MLR, Rodríguez ADL, de la Rosa APB. 2007. A novel binary expression vector for production of human IL-10 in Escherichia coli and Bifidobacterium longum. Biotechnol Lett 29: 12491253.

30. Shkoporov AN, Efimov BA, Khokhlova EV, Kafarskaia LI, Smeianov VV. 2008. Production of human basic fibroblast growth factor (FGF-2) in Bifidobacterium breve using a series of novel expression/secretion vectors. Biotechnol Lett 30: 1983-1988.

31. Takata T, Shirakawa T, Kawasaki Y, Kinoshita S, Gotoh A, Kano Y, Kawabata M. 2006. Genetically engineered Bifidobacterium animalis expressing the Salmonella flagellin gene for the mucosal immunization in a mouse model. J Gene Med 8: 13411346.

32. Yamamoto S, Wada J, Katayama $\mathrm{T}$, Jikimoto $\mathrm{T}$, Nakamura M, Kinoshita S, Lee KM, Kawabata M, Shirakawa T. 2010. Genetically modified Bifidobacterium displaying Salmonella-antigen protects mice from lethal challenge of Salmonella Typhimurium in a murine typhoid fever model. Vaccine 28: 6684-6691.

33. Suzuki R, Wada J, Katayama T, Fushinobu S, Wakagi T, Shoun H, Sugimoto H, Tanaka A, Kumagai H, Ashida H, Kitaoka M, Yamamoto K. 2008. Structural and thermodynamic analyses of solute-binding protein from Bifidobacterium longum specific for core 1 disaccharide and lacto- $N$-biose I. J Biol Chem 283: 13165-13173.

34. Katayama T, Fujita K, Yamamoto K. 2005. Novel bifidobacterial glycosidases acting on sugar chains of mucin glycoproteins. J Biosci Bioeng 99: 457-465.

35. Kitaoka M, Katayama T, Yamamoto K. 2011. Metabolic pathway of human milk oligosaccharides in bifidobacteria. In Lactic acid bacteria and bifidobacteria: Current progress in advanced research, Sonomoto K, Yokota A (eds), Caister Academic Press, 
Norfolk, pp. 53-65.

36. Ashida H, Miyake A, Kiyohara M, Wada J, Yoshida E, Kumagai H, Katayama T, Yamamoto K. 2009. Two distinct $\alpha$-L-fucosidases from Bifidobacterium bifidum are essential for the utilization of fucosylated milk oligosaccharides and glycoconjugates. Glycobiology 19: 1010-1017.

37. Kiyohara M, Tanigawa K, Chaiwangsri T, Katayama T, Ashida H, Yamamoto K. 2011. An exo- $\alpha$-sialidase from bifidobacteria involved in the degradation of sialyloligosaccharides in human milk and intestinal glycoconjugates. Glycobiology 21: 437-447.

38. Schell MA, Karmirantzou M, Snel B, Vilanova D, Berger B, Pessi G, Zwahlen MC, Desiere F, Bork P, Delley M, Pridmore RD, Arigoni F. 2002. The genome sequence of Bifidobacterium longum reflects its adaptation to the human gastrointestinal tract. Proc Natl Acad Sci USA 99: 14422-14427.

39. Ventura M, O'Flaherty S, Claesson MJ, Turroni F, Klaenhammer TR, van Sinderen D, O'Toole PW. 2009. Genome-scale analyses of health-promoting bacteria: probiogenomics. Nat Rev Microbiol 7: 6171.

40. Ventura M, Turroni F, Bottacini F, Foroni E, van Sinderen D. 2010. Genomics of bifidobacteria: where we stand now and where we are going. In Bifidobacteria: Genomics and molecular aspects. Mayo B, van Sinderen D (eds), Caister Academic Press, Norfolk, pp. 17-30.

41. Lee JH, O’Sullivan DJ. 2010. Genomic insights into bifidobacteria. Microbiol Mol Biol Rev 74: 378-416.

42. Fukuda S, Toh H, Hase K, Oshima K, Nakanishi Y, Yoshimura K, Tobe T, Clarke JM, Topping DL, Suzuki T, Taylor TD, Itoh K, Kikuchi J, Morita H, Hattori M, Ohno H. 2011. Bifidobacteria can protect from enteropathogenic infection through production of acetate. Nature 469: 543-547.

43. Sato T, Shirasawa Y, Kiwaki M, Sato S. 2004. Genome analysis of lactic acid bacteria and bifidobacteria: genome analysis of Lactobacillus casei and Bifidobacterium breve. J Intest Microbiol 18: 135-140 (in Japanese).

44. Namba N, Hatano M, Yaeshima T, Ishibashi N, Tamura Y. 2005. Whole-genome analysis of Bifidobacterium longum BB536, Bifidobacterium breve M-16V and Bifidobacterium infantis M-63. In Proceedings 8th Symposium on lactic acid bacteria, Egmond aan Zee, The Netherlands. pp. L 016.

45. Yasui K, Tabata M, Yamada S, Abe T, Ikemura T, Osawa R, Suzuki T. 2009. Intra-species diversity between seven Bifidobacterium adolescentis strains identified by genome-wide tiling array analysis. Biosci Biotechnol Biochem 73: 1422-1424.

46. Iguchi A, Umekawa $\mathrm{N}$, Maegawa $\mathrm{T}$, Tsuruta $\mathrm{H}$,
Odamaki T, Xiao JZ, Osawa R. 2011. Polymorphism and distribution of putative cell-surface adhesinencoding ORFs among human fecal isolates of Bifidobacterium longum subsp. longum. Antonie van Leeuwenhoek 99: 457-471.

47. Ishikawa E, Shima T, Suda K, Shirasawa Y, Sato T, Umesaki Y. 2011. Comparison of Bifidobacterium breve strain Yakult transcriptomes in germ-free mice with those in fecal cultures. J Biosci Bioeng 112: 451457.

48. Álvarez Martín P, Guglielmetti S, Mayo B. 2010. Mobile genetic elements, cloning vectors and genetic manipulation of bifidobacteria. In Bifidobacteria: Genomics and molecular aspects. Mayo B, van Sinderen D (eds), Caister Academic Press, Norfolk, pp. 235-259.

49. Fukiya S, Suzuki T, Kano Y, Yokota A. 2011. Current status of Bifidobacterium gene manipulation technologies. In Lactic acid bacteria and bifidobacteria: Current progress in advanced research, Sonomoto K, Yokota A (eds), Caister Academic Press, Norfolk, pp. 33-51.

50. Rossi M, Brigidi P, Matteuzzi D. 1998. Improved cloning vectors for Bifidobacterium spp. Lett Appl Microbiol 26: 101-104.

51. Shkoporov AN, Efimov BA, Khokhlova EV, Steele JL, Kafarskaia LI, Smeianov VV. 2008. Characterization of plasmids from human infant Bifidobacterium strains: Sequence analysis and construction of E. coliBifidobacterium shuttle vectors. Plasmid 60: 136-148.

52. O’Connell Motherway M, O'Driscoll J, Fitzgerald GF, van Sinderen D. 2009. Overcoming the restriction barrier to plasmid transformation and targeted mutagenesis in Bifidobacterium breve UCC2003. Microbial Biotechnol 2: 321-332.

53. O'Connell Motherway M, Fitzgerald GF, Neirynck S, Ryan S, Steidler L, van Sinderen D. 2008. Characterization of ApuB, an extracellular type II amylopullulanase from Bifidobacterium breve UCC2003. Appl Environ Microbiol 74: 6271-6279.

54. Pokusaeva K, O’Connell Motherway M, Zomer A, MacSharry J, Fitzgerald GF, van Sinderen D. 2011. Cellodextrin utilization by Bifidobacterium breve UCC2003. Appl Environ Microbiol 77: 1681-1690.

55. O'Connell Motherway M, Zomer A, Leahy SC, Reunanen J, Bottacini F, Claesson MJ, O’Brien F, Flynn K, Casey PG, Munoz JAM et al. 2011. Functional genome analysis of Bifidobacterium breve UCC2003 reveals type IVb tight adherence (Tad) pili as an essential and conserved host-colonization factor. Proc Natl Acad Sci USA 108: 11217-11222.

56. Arigoni F, Delley M. 2008. Genetic remodeling in Bifidobacterium. International Patent, WO 2008/ 019886A1. 